\title{
Classes de Estímulos por Bebês de até 24 Meses de Idade,
}

\author{
Graziele de Aguiar Roncato*** (1) \& Maria Stella Coutinho de Alcântara Gil (1) \\ Universidade Federal de São Carlos, São Carlos, SP, Brasil
}

\begin{abstract}
RESUMO - Neste estudo investigou-se a emergência de classes de equivalência generalizada, com inclusão de reforçadores específicos em bebês de 24 meses. Relações auditivo-visuais ( $\mathrm{AB}$ e AC) foram ensinadas, com consequências específicas (D), para 5 bebês entre 18 e 20 meses de idade. Testes de nomeação e de relações emergentes foram realizados (BC, $C B, A D$, $\mathrm{DE}$ e EB, com semelhança física dos estímulos E e B). Três participantes formaram de classes de equivalência, incluindo os reforçadores específicos (ABCD), e dois deles apresentaram nomeação consistente. Dois participantes apresentaram desempenho correspondente à equivalência generalizada. Os resultados indicaram (a) a inédita formação de classes de equivalência generalizada com inclusão de reforçadores específicos em bebês, e (b) que a nomeação pode não ser condição necessária para a formação de classes.
\end{abstract}

PALAVRAS-CHAVES: comportamento simbólico, controle de estímulos, classes de equivalência generalizada, reforçadores específicos, bebês

\section{Stimulus Classes to Infants up to 24 Months Old}

\begin{abstract}
This study assessed the emergence of generalized equivalence classes, with the inclusion of specific reinforcers, by infants up to 24 months old. Auditory-visual relations (AB and AC) were taught, with specific consequences (D), for five infants aged between 18 and 20 months. Naming and emergent relations tests (BC, CB, AD, DE and EB, with $\mathrm{B}$ and $\mathrm{E}$ stimuli physically similar). Three participants demonstrated equivalence classes formation, including specific reinforcers $(\mathrm{ABCD})$, and two of them presented consistent naming. Two participants demonstrated generalized equivalence classes. The results indicated the unprecedented formation of generalized equivalence classes with the inclusion of specific reinforcers in babies, and that naming may not be a necessary condition for the formation of classes.
\end{abstract}

KEYWORDS: symbolic behavior, stimulus control, generalized equivalence class, specific reinforcers, toddlers

A base de comportamentos complexos como pensamento e linguagem vem da possibilidade de os indivíduos agruparem os estímulos do mundo em classes, a partir de alguma relação em comum entre esses estímulos. As classes de estímulos podem ser formadas por relações de similaridade física ou de relações arbitrárias entre estímulos, ou seja, estímulos sem qualquer semelhança física (Plazas \& Cortés, 2017). As condições necessárias para o estabelecimento de classes com relações arbitrárias entre estímulos continuam a ser investigadas.

Alguns direcionamentos foram oferecidos por Sidman e Tailby (1982) ao proporem a teoria da equivalência de estímulos. No início do desenvolvimento dessa teoria, os autores postularam que as relações de equivalência se originariam a partir de discriminações condicionais.
Tais discriminações são experimentalmente produzidas, frequentemente, com o emprego do procedimento de matching-to-sample (MTS). Nesse procedimento, relações condicionais são treinadas entre pares de estímulos. $\mathrm{O}$ procedimento requer a exposição a dois conjuntos de estímulos: os estímulos-modelo (e.g., A), com a função de estabelecer o contexto para relação que será reforçada, e os estímulos de comparação (e.g., B e C). Cada estímulo modelo é pareado a um estímulo de comparação (e.g., A1B1). Assim, em uma tentativa de MTS, diante do estímulo modelo, o indivíduo deve escolher um entre dois ou mais estímulos comparação e a escolha convencionada como correta vai depender do estímulo modelo apresentado (Lantaya et al., 2018).

\footnotetext{
* Apoio: CAPES, INCT-ECCE

** Artigo derivado da tese de doutorado da primeira autora, orientado pela segunda autora

*** E-mail: grazielea_roncato@yahoo.com

n Submetido: 24/06/2019; Revisado: 12/04/2020; Aceito: 30/06/2020.
} 
O procedimento de MTS também pode favorecer o aparecimento de relações emergentes, que nunca foram ensinadas diretamente (Alonso-Álvarez \& Pérez-González, 2018; Sidman, 2000). Por analogia com a matemática, Sidman e Tailby (1982) afirmaram que para as relações condicionais serem consideradas relações de equivalência de estímulos é necessária a emergência de três propriedades: reflexibilidade, ou seja, que a relação do estímulo com ele mesmo seja verdadeira (e.g., relação AA); simetria, ou seja, que a reversibilidade da relação treinada seja verdadeira, por exemplo, se for treinada a relação $\mathrm{AB}$, a relação $\mathrm{BA}$ é demonstrada sem treino prévio; e transitividade, ou seja, que havendo o treino das relações $\mathrm{AB}$ e $\mathrm{BC}$, ocorra a demonstração da relação $\mathrm{AC}$ sem treino prévio.

A formação de classes de estímulos equivalentes é um fenômeno presente em diferentes populações humanas como adultos (Alonso-Álvarez \& Pérez-González, 2018; Beurms et al., 2017; Lantaya et al., 2018), crianças típicas (Canovas et al., 2018; Carp \& Petursdottir, 2015), pessoas com transtorno do espectro autista (Santos et al., 2017), entre outras. O conhecimento produzido com a pesquisa orientada pela teoria da equivalência de estímulos tem sido empregado no ensino de repertórios complexos como música (Hanna et al., 2016), química (César \& Moroz, 2018), habilidades matemáticas (Elias \& Angelotti, 2016), leitura (Paixão \& Assis, 2017) e relações monetárias (Magalhães et al., 2016).

Sidman (2000) estendeu a teoria argumentando que relações de equivalência resultam de contingências de reforçamento, ou seja, os estímulos são relacionados arbitrariamente devido à presença de um contexto com consequência reforçadora. Com isso, não somente os estímulos condicionais e discriminativos envolvidos na contingência poderiam constituir uma classe de estímulos, mas também as respostas e os reforçadores específicos da contingência. A inclusão de respostas específicas em classes de estímulos equivalentes não foi sistematicamente demonstrada (e.g., Shimizu, 2006; Urcuioli et al., 2006). Por outro lado, diversos estudos têm mostrado a inclusão de reforçadores específicos nas classes de estímulos, usando como linha de base a reversão de discriminações simples (Santos et al., 2017; Silveira et al., 2017) e discriminações condicionais, tanto com estudantes universitários (Assis et al., 2015; Silveira et al., 2018) como para pessoas com o transtorno do espectro autista (Varella \& de Souza, 2014).

Outra direção interessante dos estudos da área é a demonstração de classes de equivalência generalizadas. Estas são classes que se estabelecem sem treino prévio e que compreendem um estímulo totalmente novo, com semelhança física com os membros da classe, que também controla o responder do indivíduo e é substituível pelos outros estímulos da classe (Fields \& Moss, 2008). Nos estudos sobre classes de equivalência generalizada, inicialmente, classes de equivalência de estímulos são estabelecidas. Em seguida, são realizados testes de generalização, nos quais é verificado se os estímulos com semelhança física a algum membro da classe exerceriam o mesmo controle que o membro original sobre as respostas do indivíduo. Com esse tipo de metodologia, foram verificadas classes de equivalência generalizada em crianças surdas (Battaglini et al., 2013) e pré-escolares (Ayres-Pereira et al., 2018), entre outros participantes.

Como visto, há uma ampla demonstração do estabelecimento de classes de estímulos com humanos, porém a mesma robustez de resultados não foi encontrada com não humanos (Beurms et al., 2017). Uma possível explicação para a diferença entre os resultados de humanos e não humanos seria que o sucesso na formação de classes de equivalência requer a mediação da linguagem (Horne \& Lowe, 1996). Sidman (2000) propôs que estudos com indivíduos não humanos e com humanos com linguagem incipiente fossem realizados para investigar as condições necessárias para a formação de classes de equivalência. Os estudos com bebês são uma alternativa desejável para a tarefa, pois esta população está iniciando a aprendizagem do repertório verbal.

Os estudos sobre o desenvolvimento de repertórios simbólicos com bebês, entretanto, têm sido escassos. De Paula e Haydu (2010) realizaram uma revisão da literatura sobre estudos que investigaram equivalência de estímulos e identificaram que apenas $0,8 \%$ das pesquisas foram feitas com a população de até 24 meses de idade. Mais recentemente, Roncato et al., (2020) mostraram que a escassez de estudos com bebês permanece: ao realizaram um levantamento de pesquisas empíricas sobre a emergência de classes de estímulos em bebês de até 36 meses de idade, as autoras encontraram apenas 15 artigos publicados entre 1984 e 2018. Em um panorama geral, somente um estudo não obteve resultados esperados em formação de classes de estímulos com bebês dessa idade (Augustson \& Dougher, 1992). Dos estudos com resultados consistentes, a grande maioria utilizou o procedimento de MTS simultâneo com pareamentos visual-visual entre figuras (e.g., Devany et al., 1986; Pilgrim et al., 2011) ou objetos (e.g., Velázquez et al., 2018), com duas ou três comparações, enquanto os demais realizaram pareamentos auditivo-visual de palavra falada com figura ou objetos, com duas comparações (e.g., Postali et al., 2013).

Os reforçadores mais utilizados nas pesquisas com bebês encontradas por Roncato et al. (2020) foram os sociais ("isso", "muito bem", "parabéns", sorrisos e contato visual), seguidos pela oferta de brinquedos, adesivos e bolas de sabão. As consequências para erros foram o encerramento da tentativa, tela preta ou as palavras "não, não". Os testes foram realizados em extinção. O ponto de maior variabilidade entre os estudos foram os critérios de aprendizagem para atestar a estabilidade da linha de base, que variaram entre $75 \%$ e $100 \%$ de acertos, o que sugere a necessidade de flexibilidade nos critérios devido às peculiaridades desta população. Dos 15 estudos encontrados por Roncato et al. (2020), nenhum investigou a existência 
de classes de equivalência generalizada ou se reforçadores entrariam para as classes de estímulos formadas.

Diante desse cenário, o objetivo deste trabalho foi examinar a emergência de classes de equivalência generalizada em bebês de até 24 meses de idade e avaliar se reforçadores específicos passam a fazer parte das classes formadas. Estudos sobre a formação de classes de estímulos em bebês permitem verificar se esses indivíduos, com repertório verbal incipiente, replicam ou não os resultados obtidos com outras populações, o que pode contribuir para o avanço do conhecimento sobre os requisitos necessários para a formação de classes e suas possíveis aplicações.

\section{MÉTODO}

\section{Participantes}

Participaram do estudo 5 bebês, com idades entre 18 e 20 meses no início da coleta de dados e com até 24 meses de idade no final do experimento, e frequentadores de uma creche no interior de São Paulo. Os critérios de seleção foram: familiaridade com a pesquisadora, a autorização dos responsáveis por meio da assinatura do Termo de Consentimento Livre e Esclarecido e existência de repertório de ouvinte. O critério de exclusão foi score de risco para o desenvolvimento, segundo o Teste de Triagem Denver II (ver detalhes a seguir). O estudo foi submetido e aprovado pelo Comitê de Ética em Pesquisa com Seres Humanos da UFSCar (Parecer $n^{\circ}$ 510.041).

\section{Instrumentos}

O desenvolvimento global dos participantes foi avaliado pelo Teste de Triagem do Desenvolvimento Denver II, adaptado para o português (Sabatés, 2017) e pelo Inventário Portage Operacionalizado (Williams \& Aiello, 2001). Os dois instrumentos foram aplicados antes e após a coleta de dados. A caracterização dos participantes e os resultados obtidos pela aplicação dos instrumentos podem ser observados na Tabela 1. Os cinco participantes obtiveram score normal, segundo o Denver II, antes e após a coleta de dados. Com relação ao IPO, foi calculada a porcentagem de comportamentos apresentados de acordo com o que era esperado para a faixa etária de 2 anos e todos os participantes apresentaram acima dos $60 \%$ de acertos desde a primeira aplicação.

\section{Materiais e Equipamentos}

Foram utilizados uma filmadora digital Sony ${ }^{\circledR}$ Handycam DCR-SR21, um microcomputador Infowa Note W7535 para o processamento dos dados, uma mesa redonda e duas cadeiras, com tamanhos apropriados para bebês. Contou-se também com estímulos familiares, estímulos experimentais e palavras faladas pela experimentadora ao participante, apresentados na Figura 1.

Os estímulos familiares eram compostos por duas palavras faladas pela experimentadora ("auau" e "bola"), quatro objetos (dois cachorrinhos de pelúcia e duas bolas) e dois adesivos (dinossauros). Esses estímulos foram empregados no ensino da tarefa de SMTS (simultaneous matching-to-sample). Os estímulos experimentais eram compostos por: Conjunto A - duas palavras inventadas ("Bibóm"/“Lica") faladas pela experimentadora; Conjunto $\mathrm{B}$ - dois bonecos, um com características femininas (cabelo comprido e saia) e outro com características masculinas (cabelo curto e calça); Conjunto $\mathrm{C}$ - duas formas abstratas criadas com "Lego ${ }^{\circledR}$ "; Conjunto D - dois adesivos no formato de animais (girafa e vaca) e Conjunto E - dois adesivos no formato de bonecos, com caracteres femininos e masculinos.

\section{Procedimento}

O período de familiarização entre experimentadora e bebês ocorreu em encontros diários por até quatro semanas. O ensino da tarefa e a coleta de dados ocorreram em cerca de três sessões semanais (uma por dia), a depender

Tabela 1

Gênero, Idade em Meses, Escore Denver II e Porcentagem de Comportamentos Esperados para a Faixa Etária de Dois Anos pelo IPO no Início e Final dos Procedimentos para Cada Participante

\begin{tabular}{|c|c|c|c|c|c|c|c|}
\hline \multirow{2}{*}{ Participante } & \multirow{2}{*}{ Gênero } & \multicolumn{3}{|c|}{ Início } & \multicolumn{3}{|c|}{ Final } \\
\hline & & Idade & Denver & IPO & Idade & Denver & IPO \\
\hline $\mathrm{Sab}$ & $\mathrm{F}$ & 18 & Normal & $91 \%$ & 23 & Normal & $100 \%$ \\
\hline $\mathrm{Nic}$ & $\mathrm{F}$ & 19 & Normal & $61 \%$ & 24 & Normal & $97 \%$ \\
\hline Lor & $\mathrm{F}$ & 19 & Normal & $75 \%$ & 24 & Normal & $83 \%$ \\
\hline Dan & $\mathrm{M}$ & 20 & Normal & $100 \%$ & 24 & Normal & $100 \%$ \\
\hline Let & $\mathrm{F}$ & 20 & Normal & $77 \%$ & 24 & Normal & $91 \%$ \\
\hline
\end{tabular}




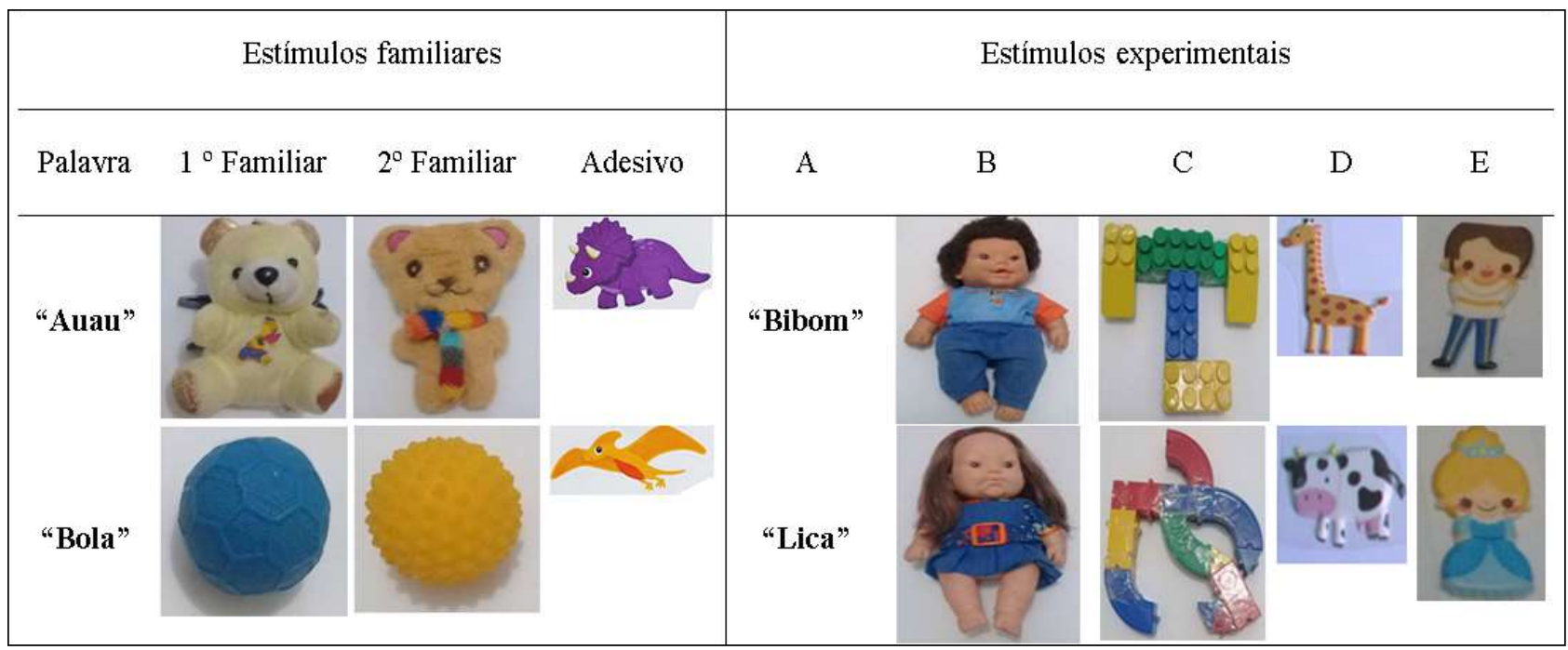

Figura 1. Conjuntos de Estímulos Familiares Usados no Ensino da Tarefa e Conjuntos de Estímulos Experimentais Usados no Ensino da Linha de Base e Testes

da disponibilidade do participante. As sessões duravam aproximadamente $10 \mathrm{~min}$, cerca de $5 \mathrm{~min}$ dispendidos nas tarefas experimentais e o tempo restante em brincadeiras livres. $\mathrm{O}$ experimento foi realizado em até cinco meses consecutivos para cada participante, compreendendo um mês de familiarização e quatro meses de ensino da tarefa e coleta de dados.

Em cada sessão, a experimentadora e o bebê sentavamse em cadeiras, frente a frente, em lados opostos da mesa. Os estímulos eram apresentados sobre a mesa, com uma distância de $25 \mathrm{~cm}$ entre eles. Ao final da sessão, eram realizadas brincadeiras livres por até $5 \mathrm{~min}$ com brinquedos diferentes dos experimentais. A brincadeira livre tinha a função de favorecer a permanência do bebê no estudo (Souza et al., 2018).

O experimento compreendeu as seguintes fases: ensino da tarefa; estabelecimentos de relações condicionais de linha de base ( $\mathrm{AB}$ e $\mathrm{AC})$; teste de nomeação 1; testes das relações emergentes (BC, CB, AD, DE e EB) e teste de nomeação 2.

Ensino da tarefa. Os participantes foram treinados com estímulos familiares, na tarefa de simultaneous matching-to-sample (SMTS). Nessa fase foram ensinadas, primeiramente, relações auditivo-visuais e, em seguida, relações visual-visuais.

A tentativa de pareamento auditivo-visual, a mesma utilizada no treino com estímulos experimentais, iniciavase com a experimentadora apresentando o modelo ao dizer "Cadê ... [a bola]?". Em seguida, a experimentadora colocava dois estímulos de comparação (bola e cachorrinho de pelúcia) sobre a mesa e reapresentava o modelo "Cadê a bola?". Em caso de não emissão da resposta de escolha (apontar ou tocar a bola) pelo participante em até $5 \mathrm{~s}$, o modelo era novamente apresentado, sendo fornecida ajuda física para o participante apontar/tocar a bola. As consequências para acerto eram (a) a retirada da comparação incorreta simultaneamente à emissão de vocalizações da experimentadora com elogios e brincadeiras, (b) brincadeira com o objeto escolhido por $5 \mathrm{~s} \mathrm{e} \mathrm{(c)} \mathrm{colagem} \mathrm{de} \mathrm{um} \mathrm{adesivo}$ específico no estímulo escolhido ( $\mathrm{S}+$ ). Caso o bebê emitisse uma resposta convencionada como incorreta, a tentativa era encerrada com a retirada das comparações e silêncio por parte da experimentadora. Alguns procedimentos de correção foram empregados: (a) em caso de preferência por posição ( $\geq 25 \%$ das escolhas em uma mesma posição), somente o estímulo de comparação correto $(\mathrm{S}+)$ era apresentado na posição contrária à posição preferida, por duas tentativas consecutivas; (b) em caso de preferência por um estímulo $(\geq$ $25 \%$ das escolhas pelo mesmo estímulo), somente o estímulo de comparação que o bebê não escolhesse era apresentado junto ao modelo por duas tentativas consecutivas; e (c) se o participante não selecionasse qualquer estímulo em até 5 sessões, os estímulos eram trocados.

A tentativa de pareamento visual-visual, similar àquela empregada nos testes com os estímulos experimentais, começava com a apresentação de um modelo visual que o participante manuseava por $2 \mathrm{~s}$; a experimentadora, então, solicitava de volta o modelo e o colocava sobre a mesa, apresentando dois estímulos de comparação, ambos também colocados sobre a mesa. Ela dizia: "Cadê o outro desse?" e apontava o modelo. As consequências para acerto e erro eram as mesmas descritas para as tentativas de ensino do pareamento auditivo-visual.

No treino de ambos os pareamentos, se o bebê emitisse três erros consecutivos, a sessão era encerrada. Cada sessão compreendeu o ensino de duas relações, sendo três tentativas para cada relação, em um total de seis tentativas. Caso o participante se engajasse em atividades concorrentes àquela de interesse da coleta ou apresentasse sinais de desconforto, a sessão era encerrada. A finalização dos treinos de cada relação ocorreu quando o critério de aprendizagem foi 
alcançado, ou seja, quando quatro acertos ocorreram em seis tentativas, por duas sessões consecutivas, desde que os erros não ocorressem para a mesma relação em uma única sessão.

Estabelecimento de relações condicionais de Linha de Base (LB). Essa fase utilizou os estímulos experimentais para ensinar as relações $\mathrm{AB}$ e $\mathrm{AC}$ pelo procedimento de SMTS com dois estímulos de comparação. Foram ensinadas primeiro as duas relações $\mathrm{AB}$ : o pareamento entre a palavra "Bibóm" (A1) e um boneco (B1), e entre a palavra "Lica" (A2) e uma boneca (B2), até que o critério de aprendizagem fosse alcançado. Em seguida, as relações AC foram ensinadas: pareamento entre a palavra "Bibóm" (A1) e uma forma abstrata (C1), e a palavra "Lica" (A2) e outra forma abstrata $(\mathrm{C} 2)$, até a obtenção de critério. Foram usadas consequências específicas para o ensino: o adesivo de girava (D1) era fornecido para as relações A1B1 e A2C2 e o adesivo de vaca (D2) era fornecido para as relações A2B2 e A2C2.

A primeira sessão de ensino tanto de $\mathrm{AB}$ como de $\mathrm{AC}$ foi composta por quatro tentativas de SMTS com uma comparação $(\mathrm{S}+)$, seguidas por duas tentativas com duas comparações ( $\mathrm{S}+$ e $\mathrm{S}-$ ). As sessões subsequentes foram compostas por seis tentativas com duas comparações ( $\mathrm{S}+$ e S-), sendo três tentativas para cada relação. Para as fases de ensino $\mathrm{AB}$ e $\mathrm{AC}$ realizadas separadamente, o critério de aprendizagem foi o mesmo da fase de ensino da tarefa.

Após o ensino de $\mathrm{AB}$ e $\mathrm{AC}$ separadamente, foram realizadas sessões de ensino misto $\mathrm{AB} / \mathrm{AC}$, compostas de oito tentativas de SMTS com dois estímulos de comparação, sendo duas tentativas para cada relação (A1B1; A2B2, $\mathrm{A} 1 \mathrm{C} 1, \mathrm{~A} 2 \mathrm{C} 2$ ). O critério de aprendizagem consistia em seis acertos em oito tentativas em duas sessões consecutivas, desde que os dois erros não ocorressem para a mesma relação em uma única sessão.

Testes de nomeação. Foram realizados dois testes de nomeação, o primeiro após o ensino da $\mathrm{LB}$ (AB e AC) e o segundo após os testes de relações emergentes $(B C$, $\mathrm{CB}, \mathrm{AD}, \mathrm{DE}, \mathrm{EB})$. A experimentadora apresentava cada estímulo visual do conjunto B e C e perguntava: "Quem é esse?", por duas vezes para cada estímulo. Se o bebê emitisse qualquer palavra, sendo a nomeação correta ou não, a experimentadora dizia "Ah!" e guardava o objeto. Não havendo nomeação, a experimentadora, sem nomear o estímulo, brincava com o bebê e o objeto por $30 \mathrm{~s}$, para identificar verbalizações espontâneas do bebê com relação ao objeto; caso o participante nomeasse o objeto, de forma correta ou não, experimentadora dizia “Ah!” e aguardava a finalização dos $30 \mathrm{~s}$ para encerrar o teste. Os testes de nomeação foram filmados e transcritos para registro das respostas orais dos participantes.

Testes de simetria e transitividade (BC e CB). Duas sessões foram realizadas em dias diferentes: uma sessão composta por seis tentativas das relações BC e outra sessão com seis tentativas das relações $\mathrm{CB}$. A programação das tentativas foi similar àquela descrita na fase de ensino da tarefa para relações visuais. Em caso de acerto, era oferecido reforçamento social e o manuseio do estímulo correto por cerca de $5 \mathrm{~s}$, sem uso do adesivo; em caso de erro, a tentativa era encerrada com a retirada de estímulos e silêncio por parte da pesquisadora. $O$ critério para demonstrar emergência das relações testadas era de acerto na primeira tentativa de cada relação e em mais uma das tentativas restantes. $\mathrm{O}$ insucesso nos testes encerrava a participação do indivíduo no estudo.

Teste AD. Esse teste verificou relações emergentes entre os estímulos experimentais auditivos (conjunto A) e os adesivos utilizados como consequência específica (conjunto D). Foi utilizado o mesmo procedimento dos testes BC e CB, ou seja, uma sessão de seis tentativas, com previsão de acerto na primeira tentativa de cada relação e em ao menos mais uma das tentativas restantes. A experimentadora apresentava a palavra modelo (A), expunha os dois adesivos do conjunto D sobre a mesa e repetia a palavra modelo.

Teste DE e EB. O teste DE verificou se os adesivos do conjunto E, que nunca haviam sido vistos pelos participantes, passaram a fazer parte das classes de estímulos formadas. $\mathrm{O}$ teste EB verificou se houve generalização entre os estímulos do conjunto B (boneca e boneco) e os E (adesivos com características masculinas e femininas) empregados no teste DE. Foi utilizado o mesmo procedimento dos testes $\mathrm{BC}, \mathrm{CB}$ e AD.

Todas as sessões foram filmadas e analisadas em termos de acerto ou erro por tentativa, computando-se o desempenho por relação. Trinta por cento das sessões filmadas foram analisadas por dois observadores independentes e foi obtido um índice de concordância de 92\% ([Concordâncias/ Concordâncias + Discordâncias] x 100).

\section{RESULTADOS}

Todos os participantes atingiram o critério com os conjuntos familiares em até 10 sessões (Sab, Lor e Dan realizaram 8 sessões; Let e Nic realizaram 10 sessões) e nomearam consistentemente os estímulos familiares de pelúcia e bolas como "auau" e "bola", respectivamente.

Com relação aos estímulos experimentais, os cinco participantes atingiram o critério de aprendizagem para a linha de base (AB/AC). A Tabela 2 sintetiza os resultados nos testes de formação de classes e de nomeação para cada participante. O desempenho de três participantes (Sab, Nic e Dan) demonstrou formação de classes de estímulos equivalentes nos testes BC e CB. O desempenho de Sab, Nic e Dan também demonstrou a inclusão dos reforçadores específicos na classe ao atingirem o critério no teste $\mathrm{AD}$. Os desempenhos de dois participantes (Nic e Dan) demonstrou a formação de classes de equivalência generalizada ao atingirem critério nos testes DE. 
Tabela 2

Alcance (Sim) ou Não do Critério de Desempenho nos Testes de Relações Emergentes e de Nomeação

\begin{tabular}{lcccccc}
\hline \multirow{2}{*}{ Participantes } & \multicolumn{3}{c}{ Testes de relações emergentes } & Testes de Nomeação \\
\cline { 2 - 6 } & BC & CB & AD & DE & EB & Primeiro / Segundo \\
\hline Sab & SIM & SIM & SIM & NÃO & NÃO & SIM / SIM \\
Nic & SIM & SIM & SIM & SIM & SIM & NÃO / NÃO \\
Dan & SIM & SIM & SIM & SIM & - & SIM / - \\
Let & NÃO & NÃO & - & - & - & NÃO / NÃO \\
Lor & NÃO & NÃO & NÃO & NÃO & SIM & SIM / SIM \\
\hline
\end{tabular}

O desempenho de cada participante será detalhado a seguir. Sab atingiu o critério de aprendizagem para as relações $\mathrm{AB}$ em 26 tentativas, para as relações $\mathrm{AC}$ em 30 tentativas e das relações mistas $\mathrm{AB} / \mathrm{AC}$ em 22 tentativas. Nos dois testes de nomeação, ela emitiu a palavra convencionada como correta perante os estímulos experimentais (conjuntos $\mathrm{B}$ e C). Sab atingiu o critério estabelecido nos testes BC, $\mathrm{CB}$ e $\mathrm{AD}$, inferindo-se que a participante formou classes de estímulos equivalentes, incluindo as consequências específicas. Sab não atingiu o critério dos testes $\mathrm{DE}$ e EB. Com isso, não houve demonstração de classes de equivalência generalizada.

Nic atingiu o critério de aprendizagem para as relações $\mathrm{AB}$ em 28 tentativas, para as relações $\mathrm{AC}$ em 22 tentativas e das relações mistas $\mathrm{AB} / \mathrm{AC}$ em 36 tentativas. Para Nic, diante da falta de respostas orais no primeiro teste de nomeação, houve a realização do teste de nomeação em situação de brincadeira; ainda assim, não houve nomeação consistente dos estímulos dos conjuntos B e C. No segundo teste de nomeação, depois dos testes de equivalência, Nic emitiu palavras não consistentes perante os estímulos. Porém, Nic atingiu o critério esperado para os testes $\mathrm{BC}, \mathrm{CB}, \mathrm{AD}$, $\mathrm{DE}$ e EB. Pode-se inferir que Nic formou duas classes de equivalência generalizadas com cinco membros, que incluíram as consequências específicas.

Dan atingiu critério de aprendizagem das relações $\mathrm{AB}$ em 30 tentativas, das relações AC em 26 tentativas e das relações mistas $\mathrm{AB} / \mathrm{AC}$ em 28 tentativas. Dan realizou apenas o primeiro teste de nomeação e acertou todas as tentativas. $\mathrm{O}$ critério dos testes $\mathrm{BC}, \mathrm{CB}, \mathrm{AD}$ e $\mathrm{DE}$ foi atingido. $\mathrm{O}$ participante deixou de frequentar a creche e não foi possível a aplicação dos testes EB e dos testes finais de nomeação. Porém, pelos resultados apresentados em DE, pode-se inferir que Dan formou duas classes de equivalência generalizadas, que incluíram as consequências específicas.

Let atingiu o critério de aprendizagem das relações $A B$ em 46 tentativas, das relações AC em 42 tentativas e das relações mistas $\mathrm{AB} / \mathrm{AC}$ em 22 tentativas, sem erros. Durante os dois testes de nomeação, Let não emitiu as palavras utilizadas no ensino. Let também não atingiu os critérios estabelecidos para os testes $\mathrm{BC}$ e CB.

A participante Lor foi exposta ao ensino das relações $\mathrm{AB}, \mathrm{AC}$ e $\mathrm{AB} / \mathrm{AC}$ mistas, porém deixou de frequentar a creche devido à uma conjuntivite. Quando a participante voltou à creche, foi reexposta aos ensinos de linha de base. Assim, Lor atingiu o critério de aprendizagem das relações $\mathrm{AB}$ em oito tentativas, das relações $\mathrm{AC}$ em 28 tentativas e das relações mistas $\mathrm{AB} / \mathrm{AC}$ mistos em 28 tentativas. Lor acertou todas as tentativas dos dois testes de nomeação para os estímulos dos conjuntos B e C. Porém, Lor não atingiu os critérios dos testes $\mathrm{BC}, \mathrm{CB}, \mathrm{AD}$ e $\mathrm{ED}$. Com isso, não houve indicações de formação de classes de equivalência de estímulos, mesmo com a nomeação dos estímulos visuais. Lor atingiu critério para as relações EB, demonstrando generalização entre os estímulos $\mathrm{B}$ (boneco e boneca) e $\mathrm{E}$ (príncipe e princesa).

\section{DISCUSSÃO}

O objetivo deste trabalho foi verificar a emergência de classes de equivalência generalizadas e inclusão de reforçadores específicos nas classes por bebês de até 24 meses. Os cinco participantes atingiram critério de aprendizagem para as quatro discriminações condicionais auditivo-visuais ensinadas e três apresentaram desempenhos condizentes com a formação de duas classes de equivalência de estímulos, incluindo os reforçadores específicos. Dos três participantes que formaram classes, somente dois nomearam consistentemente os estímulos em testes de nomeação; um participante não demonstrou nomeação consistente, o que pode indicar que a nomeação não é condição necessária para a formação de classes. Além disso, dentre esses três participantes, dois tiveram desempenhos indicando que tais classes foram expandidas com a introdução de novos membros que apresentavam similaridade física com membros pré-existentes, o que sugere a existência de classes de equivalência generalizada em bebês de até 24 meses de idade.

Com relação ao estabelecimento de linha de base, a literatura retrata variabilidade na formação de classes de estímulos em crianças pré-escolares (Carp \& Petursdottir, 
2015). A maioria dos estudos sobre formação de classes de estímulos equivalentes com bebês utiliza pareamentos visuais no ensino de discriminações condicionais (e.g., Devany, et al., 1986; Pelaez et al., 2000; Boelens et al., 2000; Jordan et al., 2001; Pilgrim et al., 2011; Postalli et al., 2013; Velázquez et al., 2018). No entanto, Boelens (2002) argumenta que relações auditivo-visuais são mais próximas ao cotidiano de crianças pequenas e devem ser alvos de investigação na aquisição de comportamentos simbólicos. Os resultados do presente estudo apoiam as afirmações de Boelens ao evidenciarem que bebês de até 24 meses aprendem satisfatoriamente a linha de base com relações auditivo-visuais.

O uso de brinquedos (bonecos com aspectos masculinos e femininos do conjunto B), similares aos do cotidiano dos bebês, pode ter contribuído para a emergência de novas relações. Pascoto (2006) indica que crianças desde antes dos três anos de idade classificam os objetos de seu meio e apresentam preferência em função do gênero. Alguns pesquisadores (Arntzen \& Nartey, 2018; Arntzen et al., 2018) argumentam que estímulos significativos, ou seja, semelhantes aos encontrados no cotidiano dos participantes, podem melhorar a emergência de classes de estímulos quando acrescentados ao ensino de relações arbitrárias. Pascoto (2006) destaca que estímulos sem sentido, como o conjunto $\mathrm{C}$ empregado neste trabalho, podem adquirir o mesmo sentido dos estímulos com sentido para o participante durante o pareamento e, assim, facilitar a formação de classes entre estímulos com e sem sentido. Com isso, esse recurso metodológico de parear estímulos familiares aos participantes com estímulos abstratos pode ser importante para o ensino de repertórios simbólicos para bebês.

Para Sab, Nic e Dan, relações emergentes foram verificadas entre a classe de estímulos e as consequências específicas. Esses resultados indicam que, mesmo com bebês de até 24 meses de idade, reforçadores específicos podem entrar para as classes de estímulos, conforme proposto pela teoria de equivalência de estímulos (Sidman, 2000). Até então, a literatura não apresentava relatos de que bebês de até 24 meses poderiam demonstrar essas relações.

Indo além, este foi o primeiro trabalho a demonstrar que bebês podem apresentar classes de equivalência generalizada. Nic e Dan, ao atingirem o critério de desempenho nos testes de expansão de classes por generalização (DE), demonstraram que houve generalização entre os estímulos novos (E) e os estímulos do conjunto B, provavelmente porque os estímulos de ambos conjuntos tinham características físicas semelhantes (Elias \& Angelotti, 2016). Assim, Nic e Dan demonstraram, logo na primeira tentativa de teste das relações entre estímulos novos e membros da classe, a formação de duas classes de cinco membros (A1B1C1D1E1 e A2B2C2D2E2). Obviamente que os estudos sobre classes de equivalência generalizada testam a associação entre o estímulo novo com todos os membros da classe existente por meio de diversos testes de transitividade. Porém, como já observado por Ayres-Pereira et al. (2018), devido às peculiaridades desta população tais como a rápida saciedade dos estímulos reforçadores e o pouco tempo de cooperação e permanência no ambiente experimental, optou-se pela realização de poucos testes para evitar a perda do participante.

Com relação às participantes que não atingiram o critério nos testes de emergência (Lor e Let), verificou-se que as linhas de base foram estabelecidas satisfatoriamente, mas isso não foi suficiente para a emergência de novas classes. Let não demonstrou nomeação consistente com as palavras do Conjunto A para os estímulos visuais dos conjuntos B e $C$, o que poderia sugerir que a ausência de nomeação foi determinante para o insucesso nos testes. No entanto, Lor demonstrou nomeação consistente dos estímulos visuais dos conjuntos $\mathrm{B}$ e C desde o primeiro teste de nomeação, mas um responder inconsistente nos testes de equivalência.

Além disso, a participante Nic não apresentou palavras consistentes para os estímulos visuais dos conjuntos B e $\mathrm{C}$ nos testes de nomeação e, ainda assim, demonstrou emergência de classes de estímulos. Com isso, os resultados de Nic sugerem que a nomeação dos estímulos não é condição necessária para a formação de classes de equivalência e os resultados de Lor indicam que a nomeação não foi suficiente para a formação de classes. Os resultados apresentados por Nic e Lor abrem margem à suposição de que a nomeação pode não ser um requisito indispensável para a equivalência de estímulos (Carp \& Petursdottir, 2015).

Existem argumentos de que os testes de nomeação podem falhar em verificar a nomeação de estímulos visuais, pois pode haver, por exemplo, nomeação encoberta (Horne \& Lowe, 1996; Carp \& Petursdottir, 2015). Entretanto, o teste de nomeação proposto neste trabalho tem sido usado em diversas pesquisas na área (e.g., Lowe et al., 2002; Luciano et al., 2007; Sidman \& Tailby, 1982). Considerando, ainda, que que os participantes deste trabalho estavam no início da aquisição verbal, parece improvável que tenha existido nomeação encoberta ou a atribuição de outros nomes aos estímulos experimentais, embora tais suposições devam ser melhor investigadas em trabalhos futuros

Ainda sobre o insucesso na formação de classes por Let e Lor, pode-se hipotetizar que a utilização dos mesmos adesivos como consequências específicas durante todas as fases da coleta de dados pode ter enfraquecido o valor reforçador desses estímulos. Santos et al. (2017) dicutiram que a perda do valor reforçador das consequências pode culminar em ampla variabilidade no desempenho intraparticipante. Cabe salientar que, neste estudo, o valor reforçador das consequências foi inferido previamente à realização do experimento, ou seja, não foi realizada uma avaliação da preferência de reforçadores antes da coleta de dados (Escobal et al., 2010), o que pode ser investigado em estudos futuros.

Entende-se que este trabalho teve algumas limitações metodológicas, por exemplo, a fragilidade de atestar 
resultados com a utilização de apenas dois estímulos de comparação (Sidman, 2000), o que possibilitaria ao participante responder ao acaso. Porém, Boelens (2002) argumenta que o uso de duas comparações pode ser uma estratégia interessante para o estudo desta população e que a análise da quantidade de acertos para cada relação ensinada, ao invés de uma análise geral de erros/acertos, contorna as dúvidas sobre o acaso na aprendizagem. Além disso, a literatura da área tem reportado resultados positivos em classes de estímulos por bebês com o uso de duas comparações (Boelens et al., 2000; Devany et al., 1986; Pelaez et al., 2000; Velázquez et al., 2018).

Entende-se, também, que o critério de aprendizagem para o estabelecimento de linha de base e para atestar o sucesso nos testes foi frágil. Mas, considerando a acelerada mudança de repertório dos participantes e as dificuldades com o estabelecimento das relações de linha de base, que também foram reportadas com participantes da mesma faixa-etária (e.g., Souza et al., 2018), pode-se considerar que é necessária maior flexibilidade no estabelecimento de critérios para esta população.

Além disso, os testes poderiam ser realizados em extinção para evitar que houvesse o ensino direto das relações emergentes. Porém, Dias e Barros (2017) não identificaram diferenças nos resultados sobre formação de classes em crianças com transtorno do espectro autista ao empregarem testes com reforçamento contínuo e testes com reforçamento intermitente, este com a primeira tentativa em extinção. Os autores afirmam que é possível haver confiabilidade em testes com reforçamento contínuo uma vez que, ao se comparar o alto número de tentativas necessárias para a aquisição das relações ensinadas com a prontidão de acertos observados nos testes dos participantes, pode-se afirmar a existência de desempenho emergente. Além disso, os testes com reforçamento contínuo facilitam a permanência das crianças com desenvolvimento atípico nas tarefas de testes.

Em suma, o presente estudo contribuiu para descrever a emergência de relações entre estímulos com indivíduos com linguagem incipiente, indicando que a nomeação pode não ser suficiente para o estabelecimento dessas relações. A despeito das limitações, a investigação demonstrou a formação de classes de equivalência generalizadas com a introdução dos reforçadores nestas classes em bebês de até 24 meses de idade.

É recomendável a replicação direta do presente estudo com um maior número de participantes e com bebês mais jovens para ampliar e detalhar os achados deste trabalho. Também seria interessante a formulação de novas pesquisas sobre os requisitos para emergência de classes de equivalência de estímulos em bebês, ou seja, pesquisas que investigassem a inclusão de respostas comuns como membros das classes e a formação de classes a partir de reversões de discriminações simples, entre outros. A área seria fortalecida pela proposição de procedimentos que auxiliassem a permanência do participante em ambiente experimental, o que aumentaria o rigor metodológico e promoveria a documentação da precocidade da formação de classes de estímulos em bebês.

\section{REFERÊNCIAS}

Alonso-Àlvarez, B., \& Pérez-González, L. A. (2018). Analysis of apparent demonstrations of responding in accordance with relational frames of sameness and opposition. Journal of the Experimental Analysis of Behavior, 110(2), 213-228. doi. org/10.1002/jeab.458

Arntzen, E., \& Nartey, R. K. (2018). Equivalence class formation as a function of preliminary training with pictorial stimuli. Journal of the Experimental Analysis of Behavior, 11(2), 275-291. doi. org/10.1002/jeab.466

Arntzen, E., Nartey, R. K., \& Fields, L. (2018). Reorganization of equivalence classes: Effects of preliminary training and meaningful stimuli. Journal of the Experimental Analysis of Behavior, 109(3), 564-586. doi.org/10.1002/jeab.329

Augustson, K. G., \& Dougher, M. L. (1992). Teaching conditional discrimination to young children: Some methodological successes and failures. Experimental Analysis of Human Behavior Bulletin, 9, 21-24.

Assis, G. J. A., Mendes, C. M., \& Prado, P. S. (2015). Efeito de reforçadores condicionados específicos em classes ordinais em humanos. Temas em Psicologia, 23(1), 211-224. https://www. redalyc.org/pdf/5137/513751490015.pdf

Ayres-Pereira, V., Canovas, D. S., Varella, A. A. B., \& de Souza, D. G. (2018). Generalization of equivalence relations from photos to objects by preschool children. Journal of the Experimental
Analysis of Behavior, 109(2), 394-407. doi.org/10.1002/ jeab.313

Battaglini, M. P., Verdu, A. C. M. A., \& Bevilacqua, M. C. (2013). Aprendizagem via exclusão e formação de classes de equivalência em crianças com deficiência auditiva e implante coclear. Acta Comportamentalia, 21(1), 20-35. https://www. redalyc.org/pdf/2745/274526149004.pdf

Beurms, S., Traets, F., Houwer, J. D., \& Beckers, T. (2017). Symmetry and stimulus class formation in humans: Control by temporal location in a successive matching task. Journal of the Experimental Analysis of Behavior, 108(3), 335-350. doi. org/10.1002/jeab. 282

Boelens, H. H. (2002). Studying stimulus equivalence: Defense of the two-choice procedure. The Psychological Record, 52(3), 305-314. doi.org/10.1007/BF03395432

Boelens, H., Broek M. V. D., \& Klarenbosch, T. V. (2000). Symmetric matching to sample in 2-year-old children. The Psychological Record, 50(2), 293-304. doi.org/10.1007/ BF03395357

Canovas, D. S., Debert, P., \& Miguel, C. (2018). Simple discrimination training with differential responses to establish functional and equivalence classes with preschool children. Journal of Experimental Analysis of Behavior, 111(1), 59-74. doi.org/10.1002/jeab.487 
Carp, C. L., \& Petursdottir, A. I. (2015). Intraverbal naming and equivalence class formation in children. Journal of the Experimental Analysis of Behavior, 104(3), 223-240. doi. org/10.1002/jeab.183

César, M. A., \& Moroz, M. (2018). Teaching chemistry and stimulus equivalence. Paidéia, 28, e2838. doi.org/10.1590/1982$4327 \mathrm{e} 2838$

de Paula, J. B. C., \& Haydu, V. B. (2010). Revisão bibliográfica de pesquisas brasileiras sobre equivalências de estímulos. Psicologia: Teoria e Pesquisa, 26(2), 281-294. https://www. scielo.br/pdf/ptp/v26n2/a10v26n2.pdf

Devany, J. M., Hayes, S. C., \& Nelson, R. (1986). Equivalence class formation in language-able and language-disable children. Journal of the Experimental Analysis of Behavior, 46(3), 243 257. doi.org/10.1901/jeab.1986.46-243

Dias, K. K., \& Barros, R. S. (2017). Classes funcionais e de equivalência em crianças diagnosticadas com autismo. Revista Brasileira de Terapia Comportamental e Cognitiva, 19(1), 18-30. doi.org/10.31505/rbtcc.v19i1.947

Elias, N. C., \& Angelotti, V. (2016). Ensino informatizado de frações para crianças surdas e ouvintes. Acta Comportamentalia: Revista Latina de Análisis de Comportamiento, 24(3), 347-363. https://www.redalyc.org/pdf/2745/274546929007.pdf

Escobal, G., Macedo, M. Z., Duque, A. L. R. F., Gamba, J., \& Goyos, A. C. N. (2010). Contribuições do paradigma de escolha para identificação de preferências por consequências reforçadoras. Em M. M. C. Hübner, M. R. Garcia, P. R. Abreu, E. N. P. de Cillo, \& P. B. Faleiros (Eds.), Sobre comportamento e cognição (361-376). São Paulo: ESETec editores associados.

Fields, L., \& Moss, P. (2008). Formation of partially and fully elaborated generalized equivalence classes. Journal of the Experimental Analysis of Behavior, 90(2), 135-168. doi. org/10.1901/jeab.2008.90-135

Hanna, E. S., Batitucci, S., \& Natalino-Rangel, P. C. (2016). Paradigma de equivalência de estímulos norteando o ensino de rudimentos de leitura musical. Acta Comportamentalia, 24(1), 29-46. https://www.redalyc.org/pdf/2745/274544251003.pdf

Horne, P. J., \& Lowe, C. F. (1996). On the origins of naming and other symbolic behavior. Journal of the Experimental Analysis of Behavior, 65(1), 185-241. doi.org/10.1901/jeab.1996.65-185

Jordan C. R., Pilgrim C., \& Galizio M. (2001). Conditional discrimination and stimulus equivalence in young children following three different baseline-training procedures. Experimental Analysis of Human Behavior Bulletin, 19, 3-7. https://pdfs.semanticscholar.org/1b9d/a97e3bf0bf0cc89570f2 ac0982965bb7568d.pdf

Lantaya, C. A., Miguel, C. F., Howland, T. G., LaFrance, D. L., \& Page, S. V. (2018). An evaluation of a visual-visual successive matching-to-sample procedure to establish equivalence classes in adults. Journal of the Experimental Analysis of Behavior, 109(3), 533-550. doi.org/10.1002/jeab.326

Lowe, C. F., Horne, P. J., Harris, F. D., \& Randle, V. R. (2002). Naming and categorization in young children: Vocal tact training. Journal of the Experimental Analysis of Behavior, 78(3), 527-549. doi: 10.1901/jeab.2002.78-527

Luciano, C., Becerra, I. G., \& Valverde, M. R. (2007). The role of multiple-exemplar training and naming in establishing derived equivalence in an infant. Journal of the Experimental Analysis of Behavior, 87(3), 349-365. doi: 10.1901/jeab.2007.08-06

Magalhães, P. G., Assis, G. A., \& Rossit, R. A. (2016). Emergência de relações monetárias por meio do procedimento de ensino de escolha com o modelo com resposta construída para crianças surdas. Revista Brasileira de Terapia Comportamental e Cognitiva, 18(2), 35-55. doi.org/10.31505/rbtcc.v18i2.882
Paixão, G. M., \& Assis, G. J. A. (2017). Uso do procedimento de Constructed Response Matching to Sample: Uma revisão da literatura. Revista Perspectivas, 8(1), 47-60. doi.org/10.18761/ pac. 2016.038

Pascoto, R. (2006). Primeiras manifestações de identidade de gênero: Um estudo com crianças de 16 a 18 meses (Dissertação de Mestrado). Pontifícia Universidade Católica de São Paulo, São Paulo, SP. https://tede2.pucsp.br/handle/handle/16293

Pelaez, M., Gewirtz, J. L., Sanchez, A., \& Mahabir, N. M. (2000, Spring). Exploring stimulus equivalence formation in infants. Behavioral Development Bulletin, 9(1), 20-25. http://dx.doi. org $/ 10.1037 / \mathrm{h} 0100534$

Pilgrim, C., Click, R., \& Galizio, M. (2011). A developmental analysis of children's equivalence-class formation and disruption. Acta de Investigación Psicológica, 1(1), 55-76. https://www.redalyc.org/pdf/3589/358933570006.pdf

Plazas, E. A., \& Cortés, D. (2017). Relation between exclusion and stimulus equivalence class formation in auditory-visual and visual-visual matching in preschoolers. International Journal of Comparative Psychology, 30(0), 1-20. https://escholarship. org/uc/item/48c2f95q

Postalli, L. M. M., Nakachima, R. Y., Schmidt, A., \& de Souza, D. G. (2013). Controle instrucional e classes de estímulos equivalentes que incluem verbos e ações. Psicologia: Reflexão e Crítica, 26(1), 136-150. doi.org/10.1590/S010279722013000100015

Roncato, G. A., Almeida, C. G. M., \& Gil, M. S. C. A. (2020). Emergência de classes de estímulos por bebês: Uma revisão sistemática de estudos empíricos. Acta Comportamentalia, 28(1), 91-108. http://revistas.unam.mx/index.php/acom/article/ view/75183/66557

Sabatés, A. L. (2017). Denver II. Teste de triagem do desenvolvimento: Manual de treinamento ( $1^{\mathrm{a}}$ ed). Lisboa: Editora Hogrefe.

Santos, E. A. L., Nogueira, C. B., Queiroz, L. L. D., \& Barros, R. D. S. (2017). Equivalence class formation via classspecific consequences in children diagnosed with autism spectrum disorder. Temas em Psicologia, 25(2), 831-842. doi. org/10.9788/TP2017.2-20

Shimizu, H. (2006). Testing response-stimulus equivalence relations using differential responses as a sample. Journal of the Experimental Analysis of Behavior, 86(2), 239-251. doi. org/10.1901/jeab.2006.04-03

Sidman, M. (2000). Equivalence relations and the reinforcement contingency. Journal of the Experimental Analysis of Behavior, 74(1), 127-46. doi.org/10.1901/jeab.2000.74-127

Sidman, M., \& Tailby, W. (1982). Conditional discrimination vs. matching to sample: An expansion of the testing paradigm. Journal of the Experimental Analysis of Behavior, 37(1), 5-22. doi.org/10.1901/jeab.1982.37-5

Silveira, M. V., Mackay, H. A., \& de Rose, J. C. (2018). Estabelecimento e reorganização de relações arbitrárias derivadas após o treino em matching-to-sample de identidade com consequências específicas. Revista Brasileira de Análise do Comportamento, 13(2), 1-18. doi.org/10.18542/rebac. v13i2.5901

Silveira, M., dos Santos, A., \& de Rose, J. (2017) Emergência de relações equivalentes a partir do treino de discriminações simples simultânea com estímulos compostos. Perspectivas em Análise do Comportamento, 7(2), 303-318. doi.org/10.18761/ pac. 2016.025

Souza, L. M. R., Gil, M. S. C. A., \& Garcia, L. T. (2018). Sondas de discriminação na avaliação da aprendizagem de relações emergentes nome-objeto por exclusão. Temas em Psicologia, 26(4), 2231-2248. doi.org/10.9788/TP2018.4-19Pt 
Urcuioli, P.J., Lionello-DeNolf, K., Michalek, S., \& Vasconcelos, M. (2006). Some tests of response membership in acquired equivalence classes. Journal of the Experimental Analysis of Behavior, 86(1), 81-107. doi.org/10.1901/jeab.2006.52-05

Varella, A. A. B., \& de Souza, D. G. (2014). Emergence of auditoryvisual relations from a visual-visual baseline with auditoryspecific consequences in individuals with autism. Journal of the Experimental Analysis of Behavior, 102(1), 139-149. doi. org/10.1002/jeab.93
Velázquez, A. G. Q., Vargas, M. A. P. V, \& Aguirre, J. F. (2018) Equivalence class formation in 11-month-old pre-linguistic infants. Acta Colombiana de Psicología, 21(1), 271-279. https:/actacolombianapsicologia.ucatolica.edu.co/article/ view/1397/1705

Williams, L. A. C., \& Aiello, L. R. (2001). O Inventário Portage Operacionalizado: Intervenção com famílias. São Paulo: Memnon. 